Reaktion. Die Spallprodukte diffundieren aus der Oberfläche, und unter günstigen Bedingungen wird das Fermentsystem wieder zum ursprünglichen Zustand regeneriert. Bei dieser Vorstellung des enzymatischen Geschehens beberrscht also die Adsorption mit ihren energetischen Folgeerscheinungen den Vorgang. Deshalb versuchte Fodor auch die Kinetik solcher in ausgesprochen kolloidem Medium verlaufenden enzymatischen Prozesse unter Berücksichtikung der Adsorption darzustellen. Die allgemeine Form des kinetischen Verlaufes läßt sich durch die Formel $\frac{d x}{d t}=k(a-x)^{n}$ darstellen. Sie bringt zum Ausdrucke, daß bei zunebmender Konzentrationsverminderung des Substrates während der Reaktion relativ mehr an das Fermentkolloid adsorbiert und damit gespalten wird. Durch das Dazwischentreten eines Diffusionsvorganges und eines Gleichgewichtsprozesses von der Form $f(a-x)$ kann somit das Bild eines rein monomolekularen Vorganges so beeinfluBt werden, dat er das Aussehen einer positiven Autokatalyse annehmen kann, ohne jedoch mit dieser irgendeine Wesensgemeinschaft zu besitzen. Für die Spaltung der Amylose durch Amylase konnten $H$. Lüers und $W$. Wasmund die Kinetik der Reaktion ebenfalls durch die von Fodor angegebene Gleichung $\frac{d x}{d t}=k(a-x)^{n}$ darstellen.

Mit der Annahme der Kolloidnatur des Fermentkomplexes und der Variabilität des kolloiden Zustandes versteht es sich von selbst, daß z. B. das Optimum der Wasserstoffionenkonzentration für die betreffende Fermentwirkung je nach den sonst noth vorliegenden Verhältnissen, z. B. dem Gehalt an anderen Ionen, ein verschiedenes und wechselndes sein kann. Abderhalden nnd Fodor haben dies an der Hefeprotease, A. Hahn und seine Mitarbeiter an der Amylaso bestätigt.

Die Befunde H. v. E ulers an den bisher reinsten Invertasepräparaten lassen dieses Enzym als einen nicht allzu kompliziert und hochmolekular zusammengesetzten Korper von Mol.-Gew, rund 20000 erscheinen. Nichtsdestoweniger aber existieren zahlreiche Fermente die sich durch ausgesprochen kolloiden Charakter auszeichnen. Ent weder kommt er ihnen als Ferment selbst zu, oder aber das vielleicht einfarh gebaute Enzym ist mit einem Kolloid zwangsläutig verbunden und macht alle Alterationen des Kolloids unter ÄuBerungen einer veränderten Wirksamkeit mit. In dieser Beziebung ist auch die Frage nach der Antigennatur der Enzyme und der Moglichkeit der Bildung spezifischer Anlienzyme im Tierkorper von Interesse. Die älteren Versuche lassen daruber keine Entscheidung zu, da die Urteile vollig widersprechend sind. Eine Studie von Kraffl-Lenz aus jüngster Zeit uber Hefesaccharase, mit moderner zuverlässiger Methodik ausgefihrt, ergab keine nennenswerte Antisaccharasebildung. H. L thers und F. Albrecht erhielten kürlich durch wiederholte subkulane lnjektion hochwertiger nach Sherman dargestellter Malzamylasepräparate ein Serum von starker antiamylatischer Wirksamkeit. Die Bindung von Enzym und Antienzym folgt bis zu einer Wirkungshemmung von $75 \%$ rein stðchiometrischen Gesetzen, erst von $80 \%$ Hemmung an nahm die Menge des erforderlichen Immunserums mehr and mehr zu. Die Antiamylase war spezifisch nur auf Malzamylase wirksam, auf Speichel- und Pankreasamylase vollig unwirksam. Die hemmende Wirkung der Antiamylase kann darauf zurückgefithrt werden, dak der im Immunserum entbaltene Antieiweißkઠ̋rper mit dem Antigeneiweiß der Amylase unter Präcipitierung zu eioer Adsorption des eigentlichen Enzymes fubrt, daB also die Wirkung keine direkte, sondern eine indirekte sekundäre ist. Die Präcipitinreaktion ließ sich denn auch mit aller Deutlichkeit nachweisen. Als man nun in Gegenwart von Amylase eine Präcipitinreaktion mit Eialbumin als Antigen und seinem Immunserum hervorrief, zeigte sich nicht die geringste Hemmung des Enzymes. Daraus kann man folgern, daß entweder tatsächlich ein artspezifisches Antienzym gebildet wurde, oder, was das wabrscheinlichere ist, daß die Verkettung der Amylasefunktion mit einem Eiweißkorper des Malzes eine ganz spezifische und fïr die Wirksamkeit des Komplexes unerläBlich ist.

Es ist nicht moglich, im Rahmen eines derartig karzen Berichtes auf alle in den letzten Jahren zutage geforderten Erkenntnisse auf dem Enzymgebiet einzugehen. Aus dem Referierten gebt wobl hervor, daß die Auffassungen über diese wichtigen und geheimnisvollen Werkzenge der Iebenden Natur noch weit auseinandergehen, wie aber anderseits auf verschiedenen Wegen die Forschung dem Wesen der Enzyme näber zu kommen sucht. Zwei Meister der Chemie, Willstätter und Haber betonten in letzter Zeit, daß für dia chemisrhe Forschung nach der Vollendung der auf Destillation und Kristallisation beruhenden Methoden nunmehr die Zeit gekommen sei, einen großen Schritt vorwărts zu mächen, nämlich die Meihoden der Pflanzen nachzuabmen und bei gewöhnlicher Temperatur in wässerigem Medium mit gelinden Mitteln und den reaktionsfahigsten Atomgrupien und den feinsten katalytischen Helfern die lebenswichtigen Stoffe aufzuhauen. Dies wird aber nur dann erreichbar sein, wenn wir erst einmal uher das Wesen der Enzyme vobllig im klaren sind. Dann wird es sich auch zeigen, ob die Bildung der Enzyme ein Vorrecht des lebenden Protoplasmas ist, oder ob es möglich se $n$ wird, der organischen Materie die Funktionen des I,ebens zu verleihen. Der alte Streit zwischen der mechanistischen und vitalistischen Aufiassung wird damit vollends sein Ende finden.

\section{Die Bestimmung des Kohlenstoffs im Nickel.}

Von Dr. K. Breisch und Dr. K. ChalupNy.

Mitteilungen aus der Metallurgischen Versuchsanstalt der Berndorfer-Metallwarenfabrik A. Krupp, A.-G., Berndol ( Niederöscerrich!.

(Eingeg. 23.88. 1922.)

Vorliegende Arbeit, zu der uns unser Chef, Herr Dr. R u dolf K г IIa, die Anregung und manchen wertvollen Rat gegeben hat, wurde von uns aus folgenden Gründen durchgeführt:

Angaben über die Bestimmung des Kohlenstoffs im Nickel sind sowohl in den Zeitschriften als auch in den analytischen Lehrbüchern nur wenige und dann sebr karge zu finden.

Die bisher wohl allgemein verwendete Methode, die Bestimmung des Kohlenstoffs durch Verbrennung mit Chromschwefelsäure nach Corle is vorzunehmen, ist sehr langwierig, und außerdem sind die Materialkosten (Chemikalien, Gas und Verschleiß an Apparatur) sehr hohe.

Uber die direkte Verbrennung im elektrischen Ofen, die nicht ohne weiteres durchführbar ist, sind in der Literatur nur ungenügende Hinweise zu finden.

Die einzig ausführlichere Arbeit von $\mathrm{Orthey^{1 } )}$ schlägt als günstigste Art der Durchführung das Lösen des Nickels in einer neutralen Kupferammonchloridlösung unter Rühren, Abfiltrieren des zurückgebliebenen Kohlenstoffs auf ein Asbestfilter und Verbrennen des Rückstands im Corleis vor. Abgeseben davon, daB die von O r t h $\theta$ y angegebene Lösungsdauer von $40 \mathrm{Min}$. für die meisten Nickelsorten nicht zulrifft, und die Lösung oft ganz wesentlich längere Zeit eriordert, ist auch bei der von ihm verlangten feinsten Zerkleinerung die Gefahr sehr groß, daß die Probe durch das Feilen mit Eisen verunreinigt zur Verwendung gelangt. Ein Pulverisieren durch Zerschlagen ist ja bei Nickel unmöglich. Nach unserem Ermessen müßte eine im Betriebslaboratorium zu verwendende Methode auch mit gröberem Material, z. B. von den Dimensionen leiner Bohr- oder Hobelspäne durchfürbar sein.

Für den Gang unserer Untersuchungen war einerseits die Möglichkeit, bestehende Methoden zu beschleunigen, und in zweiter Linie das Bestreben, eine Lösung nach neuen Gesichtspunkten zu suchen, maB. gebend.

Verbrennung mit Chromschwelelsänre im Corleiskolben.

Die Bestimmung des Kohlenstoffs im Nickel durch Verbrennung mit Chromschwefelsäure im Corleiskolben leidet vor allem an der langen Dauer der Durchführung, während welcher, wenn brauchbare Resultate erzielt werden sollen, ständige Beaufsichtigung nötig ist. Da Verbesserungen an der Apparatur kaum in Betracht kommen, beschäftigen wir uns lediglich damit, die Lösungsdauer des Nickels durch entsprechende Zusammenstellung des Chromschwelelsäuregemisches herabzusetzen.

Die zur Kohlenstoffbestimmung in Eisen und Stahl verwendeten, Kupfersulfat enthaltenden Mischungen sind für Nickel völlig ungeeignet. Nach unseren Ermittlungen ist das Kupferion bei Nickel überhaupt von geringem Einfluß auf den Lösungsvorgang. Die Lösungsgeschwindigkeit nimmt mit zunehmender Schwefelsäure- und Chromsäurekonzentration $\mathrm{zu}$, dies aber auch nur bis $\mathrm{zu}$ einem gewissen Grad, da hochkonzentrierte Schwelelsäure wieder wesentlich langsamer löst, so daß z. B. mit der von $\mathrm{O}$ t h e y erwähnten Mischung von $20 \mathrm{ccm}$ Chromsäurelösung (180 g Chromsäure in $100 \mathrm{ccm}$ Wasser $)$ und $150-200 \mathrm{ccm}$ konzentrierter Schwefelsäure ein achtstundiges Kochen der Probe notwendig ist. (Auf die Gefährlichkeit dieser Mischung bei event. Springen des Kolbens sei noch aulmerksam gemacht.)

Vergleichende Versuche mit einer größeren Anzahl Chromschwefelsäuremischungen ergaben als günstigste Zusammensetzung die folgende:

$200 \mathrm{~g}$ Chromsäure in $400 \mathrm{~g}$ Wasser gelöst und mit $150 \mathrm{ccm}$ kanzentrierter Schwefelsäure versetzt. Davon werden zu einer Bestimmung $150 \mathrm{ccm}$ verwendet und jedesmal erneuert. Mit diesem Gemisch gelingt es, $5 \mathrm{~g}$ Nickel in etwa 3 Stunden restlos autzulösen. Bei einigen zu diesen Versuchen hergestellten Nickelspeziallegierungen, Silicium-, Wolfram- und Bornickel (je $1 \%$ ig) dauert die Auflösung auch bei Verwendung dieser Mischung wesentlich länger.

Bezüglich der Trocknungs- und Absorptionsapṕaralur wäre nur auf eine Einzelheit hinzuweisen. Es ist nicht angängig, die zwischen Corleiskolben und dem Verbrennungsrohr mit Kupferoxyd unbedingt nötige Trocknung durch ein Chlorcalciumrohr vorzunehmen. In diesem bilden sich infolge der nicht unbeträchtlichen Wasserdamp? mengen, welche durchpassieren, sehr bald geringe Mengen gesättigter Chlorcalciumlauge, die verhältnismäßig viel Kohlensäure absorbiert, auch dann, wenn das Chlorcalcium vorher mit Kohlensäure gesättigt war, was auch trotz jedesmaligem Wechsel zu größeren Kohlensäureverlusten führen kann. Einwandfrei verwendbar ist hier nur konzentrierte Schwefelsäure in einer Waschflasche. Als Absorptionsrohre verwendeten wir in allen Fällen U-Rohre mit Natronkalk und Chlorcalcium.

Die nach dieser Methode erhaltenen Kohlenstoffwerte können nicht unmittelbar mit nach anderen erhaltenen verglichen werden.

1) Orthey, Gießerei-Ztg. 1912, Nr. 8, S. 237. 
Einige Blindversuche, bei welchen man die vorher sorgfältig ausgekochte Chromschwefelsäure durch etwa drei Stunden in der zusammengestellten Apparatur kochen läßt, lehren dies, alle ergeben nicht unbeträchtliche Gewichtszunahmen der Absorptionsrohre, die im Mittel etwa $0,0025 \mathrm{~g}$ betragen. Die in den folgenden Abschnitten angeführten, nach der Chromschwefelsäuremethode ermittelten Vergleichszahlen sind daher nach Abziehen von $0,0025 \mathrm{~g}$ von der Gewichtszunahme der Absorptionsrohre errechnet. (Es sei gleich bemerkt, daß Blindversuche mit dem elektrischen Ofen bei gut gereinigtem Sauerstoff nur sehr geringe Gewichtszunahmen ergeben.)

Auflösen der Probe in Salpetersäure bestimmter Konzentration und Verbrennen des Rückstundes.

Wir versuchten, analog der von $L \in d e b u r$ angegebenen Methode zur Bestimmung des Graphits in Eisen und Stahl beim Nickel den Gesamtkohlenstoff ebenfalls durch Lösen der Probe in Salpetersäure und nachheriger Verbrennung zu bestimmen.

Die Annahme, daß dies möglich sei, ist damit begründet, daß wir, wie aus dem nachfolgenden Abschnitt über die anodische Auflösung hervorgeht, gefunden haben $\mathrm{da \beta}$ der gebundene Kohlenstoff im Nickel gegenüber dem Angrifi verdünnter Säuren wesentlich widerstandsfähiger ist als in Eisen.

Die von $L$ e d e b u r angegebene Salpetersäure von 1,2 spez. Gewicht löst das Nickel in der Kälte nur sehr langsam, selbst wenn man die Flüssigkeit kocht, dauert die Lösung 10-15 Minuten, wobei keine merkliche Stickoxydentwicklung auftritt. Wir versuchten zur Beschleunigung des Lösungsvorganges auch Salpetersäure etwas otärkerer Konzentration zu verwenden, kamen jedoch, gleichgültig welche Konzentration verwendet wurde, immer zu dem Resultat, daß dabei vom vorhandenen Kohlenstoff nur ein geringer Bruchteil (nur der als Graphit vorhandene) im Rückstande blieb. Eine Ausnahme davon machte nur die zu unseren Versuchen herangezogene kohlenstoffreiche Nickelsorte. Hier war der Kohlenstoff fast ausschließlich ale Graphit vorhanden. Auch Orthøy erwähnt diesen Mißerfolg ohne darauł näher einzugehen. Eine Lösung in. Salzsäure oder Schwefelsäure kommt wegen der ungemein trägen Einwirkung dieser Săuren auf Nickel nicht in Betracht.

Im folgenden einige Vergleichszahlen:

\begin{tabular}{|c|c|c|c|c|}
\hline Probematerial & $\begin{array}{c}\text { Einwage in } \\
\mathrm{g}\end{array}$ & $\begin{array}{c}\text { Auswage in } \\
\mathrm{g}\end{array}$ & $\begin{array}{c}\text { Gehalt an } \\
\mathrm{C} \text { in } \\
\%\end{array}$ & $\begin{array}{c}\text { Nach dem } \\
\text { Chromschwefel- } \\
\text { säureverfahren } \\
\text { gefunden (Mittel) } \\
\%\end{array}$ \\
\hline NiekeI II. . & $\begin{array}{l}3,024 \\
3,009 \\
3,004\end{array}$ & $\begin{array}{l}0,0052 \\
0,0058 \\
0,0050\end{array}$ & $\begin{array}{l}0,047 \\
0,452 \\
0,045\end{array}$ & 0,128 \\
\hline $\begin{array}{l}\text { Kohlenstoff . } \\
\text { Nickel. . . }\end{array}$ & $\begin{array}{l}1,0016 \\
1,0015\end{array}$ & $\begin{array}{l}0,0645 \\
0,0659\end{array}$ & $\begin{array}{l}1,76 \\
1,79\end{array}$ & 1,83 \\
\hline
\end{tabular}

Anodische Auflösung des Nickels.

a) Verbrennung des Rückstandes im elektrischen Ofen.

Die charakteristischen Einzelheiten des im folgenden beschriebenen Verfahrens wurden uns von unserem Chef, Dr. K r u l l a, mrgeteilt. Das Prinzip ist folgendes: Die auf ihren Kohlenstoffgehalt zu untersuchende Nickelprobe befindet sich in einer Platinschale, welche als Badflüssigkeit verdünnte Schwefelsäure enthält. Durch diese Anordnung wird ein elektrischer Strom geschickt, die Schale und das aut deren Boden befindliche Nickel sind dabei als Anode geschaltet. Um die Sicherheit und Geschwindigkeit der Auflögung zu erhöhen, wird ein starker Elektromagnet (massiver Eisenkern Wicklung hat $647 \Omega, 0,17 \mathrm{~A}$, direkt an die Gleichstromleitung $\mathrm{mi}$ 110 Volt angeschlossen, und zwar durch einen für Rührmotor bestimmten Steckkontakt) unter der Schale so angeordnet, daß er die Nickelteilchen fest an deren Boden anpreßt und so die Stromleitung auch durch das Nickel sicherstellt. úberdies wird während des Auflösungsprozesses noch langsam gerührt.

Es sei gleich vorausgeechickt, daß diese Methode zum Ziel führte, und die auf diesem Weg ermittelten Resultate gut mit den durch Verbrennung im Corleiskolben erhaltenen übereinstimmten, woraus sich ergibt, daß der gebundene Kohlenstoff im Nickel bei der anodischen Auflösung mit verdünnter Schwefelsäure keine Kohlenwasserstoffe entwickelt.

Die Konstruktion eines neuen Apparates zur Durchführung der Auflösung ist überflüssig. Nach einfachen Umänderungen an einem Schnellelektrolysenstativ konnten wir die anodischen Auflösungen in der eben angedeuteten Form gut vornehmen. An dem zur Verfügung stehenden $F$ is cherschen Schnellelektrolysenstativ mit biegsamer Welle wurden ersetzt: Der Becherglasbehälter durch einen Messingring mit Klemmschraube am Ansatz als Sitz und Stromzuleitung fïr die Platinschale, und der gewöhnliche Rührer durch einen unten seitlich abgebogenen flachgedrückten Glasstab. Die Schaltung des Lösungsstromes ergibt sich dann von selbst. Als Kathode benützt man am besten einen $1 \mathrm{~mm}$ starken Alpaka- oder Messingdraht (Eisen und Nickel sind, da magnetisch, unverwendbar; Platin ist unnötig); sie taucht nur ganz wenig in die Flüssigkeit ein, die hohe kathodische Stromdichte ist dabei nicht von Einfluß. Wir konnten nämlich feststellen, daß an der Kathode stets ein nicht unerheblicher Teil des abgeschiedenen Kohlenstoffs festhaftet, weshalb es unerläBlich ist, den eingetauchten Teil abzuzwicken und in das Verbrennungsschiffchen mitzugeben. Als Lösungsmittel verwendeten wir verdünnte Schwefelsäure 1:4 (spez. Gew. 1,14), und zwar $20 \mathrm{ccm}$ pro Gramm Einwage. Bei stärkerer Säurekonzentration krystallisiert leicht Nickelsulfat aus und entzieht dann häufig Nickelteilchen der Auflösung. Ist weniger als die eineinhalbfache stöchiometrisch zur Lösung nötige Menge Schwefelsäure vorhanden, so tritt gewöhnlich Bildung unlöslichen basischen Nickelsulfats auf, welches man vor dem Abfiltrieren durch längeres gelindes Erwärmen nach Zugabe von Schwefelsäure in Lösung bringen muß.

Es standen uns 7 wei Platinschalen mit einem nutzbaren Inhalt von etwa 60 und $120 \mathrm{ccm}$ zur Verfügung. In der kleineren, in welcher man bei Verwendung desselben Stromes die doppelte anodische Stromdichte gegenüber der größeren hat, ging die Lösung auch wesentlich rascher vonstatten, doch trat hier bei Auflösung einer Einwage von $5 \mathrm{~g}$, wie sie zur Kohlenstoffbestimmung im technischen Nickel mit einem Gehalt von etwa $0,1 \%$ nötig ist, sehr häułig infolge Säuremangels Bildung von basischem Nickelsulfat auf. Daraus ergibt sich, daß ein für alle Fälle sicheres Arbeiten nur mit Platinschalen von mindestens $100 \mathrm{~cm}$ Inbalt gewährleistet ist.

Wie zu erwarten, wächst mit der Steigerung der anodischen Stromdichto auch die Lösungsgeschwindigkeit. Höhere Stromstärken im gewöhnlich verwendeten Ausmaß (bis zu $\mathrm{ND}_{100}=6-7 \mathrm{~A}$ ) fuihren nach unserer Erfahrung zu keinen Kohlenstoffverlusten, so daß man wich diesbezüglich nach der Leistungsfähigkeit der zur Verfügung stehenden Stromquelle richten kann.

Um zu erreichen, daß sich die am Boden der Schale bildende spez. schwere Nickelsulfatlösung rasch mit der übrigen Flüssigkeit mischt, ist schwaches Rühren angezeigt $(60-100$ Touren pro Minute genügen). Die Schale wird wie bei der Elektrolyse mit einem ausgeschnitten Uhrglas bedeckt. Auch bei größeren Einwagen ist die P'robe bei $\mathrm{ND}_{100}=3-4 \mathrm{~A}$ in vier Stunden gewöhnlich gelöst. Zurückbleibende Partikel wägt man zurück. Nach Beendigung der Auflösung spült man den Schaleninhalt in ein Becherglas und filtriert durch einen kleinen Glastrichter, der ein Astbestfilter enthält (dieses bereitet man folgendermaßen vor: Ein kleiner Bausch reiner Glaswolle wird befeuchtet und fest in die Spitze des Trichters gedrückt, dann gießt man ausgeglühten, aufgeschlemmten, feinfaserigen Asbest darauf, so daß ein nicht zu dünnes Asbesthäutchen zurückbleibt, es müssen dann die Troplen noch in rascher Folge abrinnen). Bei richtiger Herstellung des Filters wird der ganze Rückstand vom Asbest zurückgehalten, so daß beim Überführen in das Schiffchen die Glaswolle nicht mitgegeben werden muß. Sie würde im elektrischen Ofen schmelzen und dabei Teilchen des Rückstandes einschließen. Bei manganiertem Nickel tinden sich infolge anodischer Oxydation oft größere Mengen flockigen Mangansuperoxydhydrats im Rückstand, die jedoch in keiner Weise stören. Man wäscht mit Wasser bis das Ablaufende nicht mehr sauer reagiert und überführt, wie schon erwähnt, möglichst nur Asbest und Rückstand in ein Verbrennungssehiffchen. Dieses wird etwa eine Stunde im Trockenschrank getrocknet, dann im elektrischen Olen durch zehn Minuten au 900-1000 ${ }^{\circ}$ erhitzt und nach Ausschalten des Stromes der Sauerstoff noch etwa zehn Minuten hindurchgeleilet. Sehr wichtig ist dabei, $\mathrm{da} \beta$ der Kohlenstoff nur dann den Ofen gänzlich als Kohlendioxyd verläßt, wenn die Verbrennungsgase noch in der Glühzone durch einen kurzen Zylinder aus zusammengerolltem Platindrahtnetz von der Weite des Porzellanrohres streichen, worauf wir noch zurückkommen werden. Absorptions- und Reinigungsgefäße sind dabel die gewöhnlich zu Kohlenstoffbestimmungen verwendeten. Ein U-Rohr mit trockener Chromsăure wird gleich nach dem Verbrennungsrohr eingeschaltet. Gegenüber der Verbrennung mit Chromschwefelsäure im Corleis bietet diese Methode, ganz abgesehen vom wesentlich geringeren Materialverbrauch, den Vorteil, daB eine ständige Beaufsichtigung gänzlich überflüssig ist und sie auch dadurch wesentlich sparsamer wird. Zum Vergleich seien einige so erhaltene Resultate den nach $\mathrm{Corl}$ i is ermittelten gegenübergestellt:

\begin{tabular}{|c|c|c|c|c|}
\hline Probematerial & $\begin{array}{c}\text { Einwage in } \\
\mathrm{g}\end{array}$ & $\begin{array}{c}\text { Auswage in } \\
\text { g }\end{array}$ & $\begin{array}{c}\text { Gehalt an } \\
\mathrm{C} \text { in } \\
\%\end{array}$ & $\begin{array}{c}\text { Nach dem } \\
\text { Cbromschwefel- } \\
\text { săureverfahren } \\
\text { gefunden } \\
\text { (Mittel) } \%\end{array}$ \\
\hline $\begin{array}{l}\text { Kohlenstoff . } \\
\text { Nickel. . . }\end{array}$ & $\begin{array}{l}1,0000 \\
3,0000 \\
1,0029\end{array}$ & $\begin{array}{l}0.0675 \\
0.2003 \\
0.0672\end{array}$ & $\begin{array}{l}1,84 \\
1,82 \\
1,83\end{array}$ & 1,83 \\
\hline Nickel II . . & $\begin{array}{l}3.0000 \\
3,0028\end{array}$ & $\begin{array}{l}0,0139 \\
0,0143\end{array}$ & $\begin{array}{l}0126 \\
0,130\end{array}$ & 0,128 \\
\hline Nickel Ill . . & $\begin{array}{l}5,000 \\
5,008 \\
3,383\end{array}$ & $\begin{array}{l}0,0087 \\
0,0094 \\
0,0067\end{array}$ & $\begin{array}{l}0,047 \\
0051 \\
0,048\end{array}$ & 0,050 \\
\hline $\begin{array}{l}\text { Französ. Ron- } \\
\text { dellen Nickel }\end{array}$ & $\begin{array}{l}3,000 \\
3,000\end{array}$ & $\begin{array}{l}0,0038 \\
0,0046\end{array}$ & $\begin{array}{l}0,035 \\
0,041\end{array}$ & 0,037 \\
\hline $\begin{array}{l}\text { Gehämmertes } \\
\text { Gußnickel }\end{array}$ & $\begin{array}{l}5,006 \\
5,000\end{array}$ & $\begin{array}{l}0,0152 \\
0,0160\end{array}$ & $\begin{array}{l}0,083 \\
0,087\end{array}$ & 0,088 \\
\hline
\end{tabular}


Eine recht günstige Verwendung kann dieso Methode auch bei der Bestimmung des Kohlenstoffgehaltes einiger Nickelspeziallegierungen finden, denen derzeit allerdings keine technische Wichtigkeit zukommt. Wir untersuchten daraufhin Legierungen unseres kohlenstoffreichen Nickels mit je $1 \%$ Silicium, Wolfram und Bor. Insbesondere Silicium- und Wolframnickel lösen sich in Chromschwefelsäure auberst langsam, Die anodische Auflösung geht bei allen ohne Schwierigkeit vor eich. Soweit bei der Verbrennung mit Chromschwefelsüure brauchbare Resultate erhalten werden konnten (wenn sich die Verbrennungsmischung nach der Verbrennung als rückstandfrei erwies), zeiglen diese mit den bei der anodischen Auflösung erhaltenen gute ưbereinstimmung.

\begin{tabular}{|c|c|c|c|c|}
\hline Probematerial & $\begin{array}{c}\text { Einwage in } \\
\mathrm{g}\end{array}$ & $\begin{array}{c}\text { Answage in } \\
\text { g }\end{array}$ & $\begin{array}{c}\text { Gehalt an } \\
\text { C in } \\
\%\end{array}$ & $\begin{array}{l}\text { Nach dem } \\
\text { Chromschwefel- } \\
\text { säureveriabren } \\
\text { gefunden } \\
\text { (Mittel) } \%\end{array}$ \\
\hline Siliciumnickel & $\begin{array}{l}1,0011 \\
1,0017\end{array}$ & $\begin{array}{l}0,0629 \\
0,0638\end{array}$ & $\begin{array}{l}1,72 \\
1,74\end{array}$ & 1,71 \\
\hline Wolframnickel & $\begin{array}{l}1,0014 \\
1,0023\end{array}$ & $\begin{array}{l}0,0646 \\
0,0664\end{array}$ & $\begin{array}{l}1,76 \\
1,80\end{array}$ & 1,76 \\
\hline Bornickel. . & $\begin{array}{l}1,0012 \\
1,0002\end{array}$ & $\begin{array}{l}0,0809 \\
0,0820\end{array}$ & $\begin{array}{l}2,21 \\
2,24\end{array}$ & 2,22 \\
\hline
\end{tabular}

b) Direkte Auswage des Kohlenstoffs.

Versucht man den bei der anodischen Auflösung erhaltenen Rückstand nach Abfiltrieren durch einen Goochtiegel, Waschen und Auswăgen zur Berechnung des Kohlenstolls zu verwenden, so bekommt man bedeutend zu hohe Resultate, da ein großer Teil des vorhandenen Siliciums als Kieselsäure, lerner gewöhnlich auch geringe Mengen feiner Nickelteilchen und eventuell auch Mangansuperoxydhydrat vorhanden sind. Zur Reinigung von diesen Beimengungen wurde mit heißer 10\% Die dabei erbaltenen Zahlen näherten sich den richtigen Werten wesentlich, fielen jedoch auch bei mehrmaliger Wiederholung des Waschens noch immer zu hoch aus. Wir erhielten z. B. bei Nickel mit $1,83 \%$ Kohlenstoff $2,18 \%, 2,13 \%$. Es gelang uns, in keiner Weise (auch nicht durch Waschen mit heißer Salzsäure) den richtigen Resultaten näherzukommen.

\section{Direkte Verbrennung im elektrischen Ofen.}

Eine gleichartige Durchführung wie bei der Bestimmung im Eisen und Stahl, d. h. das Nickel im Ofen uber den Schmelzpunkt etwa $1480^{\circ} \mathrm{zu}$ erhitzen, ist nicht möglich, da bei dieser Temperatur nach unserer Erfahrung Verbrennungsrohre aus den in Betracht kommendea Materialien nicht mehr sicher gasdicht sind, und auch die Heizkörper der elektrischen öfen solchen Temperaturen kaum lange standhalten.

Nimmt man die Verbrennung bei $1100-1200^{\circ}$ vor, wie für Eisen und Stahl in Lede burs Leitfaden für Eisenhüttenlaboratorien beschrieben ist, so ergibt sich nur eine ganz geringfügige Gewichtszunahme der Absorptionsröhren, das Nickel wird dạbei nur oberflächlich oxydiert und blauschwarz. Es ist also der Schiuß sehr naheliegend, daß unter diesen Umständen auch nicht annähernd gelingt, den in der Probe enthaltenen Kohlenstoff zu oxydieren.

Wir versuchten deshalb die Verbrennung unter Zuhilfenahme oxydierender Zumischsubstanzen durchzuführen und verwendeten dabei Bleisuperoxyd, Kaliumbichromat, Kaliumbichromat gemischt mit Kaliumhydrosulfat, Kobaltoxyd und schlieblich ein Gemisch von Aluminiumpulver, Aluminiumoxyd und Bleisuperoxyd. In keinem Fall wurden zufriedenstellende Ergebnisse erreicht, immer tehlte ein oft beträchtlicher Teil Kohlendioxyd.

Eine andere Erklärungsmöglichkeit fïr dieses Versagen ist die Bildung von Kohlenoxyd. Bestimmt man nach L e d e b u $\mathbf{r}$ den Kohlentoffgehalt im Eisen im elektrischen Ofen und läßt man dabei den Sauerstoffstrom ziemlich langsam durch die Apparatur gehen, so ergeben sich bedeutende Verluste durch Kohlenoxydbildung. Bei Nickel ist gwar auch dann ein größerer Sauerstoffmangel nicht denkbar, da zur Oxydation des Metalls nur ziemlich geringe Mengen verbraucht werden. Rascheres Durchleiten von Sauerstoff ändert daher in diesem Falle auch gar nichts. Daß der Kohlenstoff dabei aber doch verbrannte, war leicht nachzuweisen, inden die im elektrischen ofen geglühte Probe möglichst zerkleinert mit Chromschwefelsäure im Corleis zersetzt wurde. Es ergab sich in keinem Fall eine Gewichtszunahme der Absorptionsrohre, größer als bei Blindversuchen. Der Kohlenstoff konnte also nur als Kohlenoxyd die Apparatur verlassen, und eine nachträgliche Oxydation mußte Erfolg haben.

Führt man, wie schon trüher erwähnt, ein zusammengerolltes Platindrahtnetz in das Verbrennungsrohr so ein, daß es gerade noch in der Glühzone liegt, so erhält man Ergebnisse, die sowohl untereinander als auch mit den durch Verbrennung mit Chromschwefelsäure ermittelten völlig übereinstimmen. Die Form, in der das Nickel dabei vorliegt, ist nicht von Einfluß, z. B. wird auch aus $1-2 \mathrm{~mm}$ starken Bohrspünen der Kohlenstoff sehr rasch restlos herausgebrannt. Dieses Ergebnis bestätigt die Richtigkeit der kurzen, auf Nickel bezüg- lichen Anmerkung in $B$ a u e $-D$ e $B$, Probenahme und Analyse von Eisen und Stahl, 2. Aufl., S. 144. Die Verfasser geben für die Kohlenstoffbestimmungen im Eisen and Stahl eine Füllung des Rohres nach dem Verbrennungsschiflchen mit Kobaltoxyd in $2-6 \mathrm{~cm}$ langer Schicht innerhalb des Ofens und einem Gemisch von Kaliumbichromat und Kaliumchromat außerhalb des Ofens an. Die Verwendung des Platindrahtnetzes ist unseres Erachtens insbesondere im Betriebslaboratorium wesentlich handlicher; ob es sich auch bei Bestimmung des Kohlenstoffs in Eisen und Stahl bewährt, wäre noch festzustellen. Das von uns verwendete Drahtnetz ist von derselben Stärke wie bei Netzelektroden, quadratisch von etwa $4 \frac{1}{2} \mathrm{~cm}$ Seitenlänge; es wurde längs einer Mittellinie zusammengelegt und der Schmalseite nach gerollt. Dieser Zylinder hat die Bohrung des Verbrennungsrohres möglichst auszufüllen. Das Platinnetz erleidet bei den Verbrennungen keinerlei Verschleiß oder Veränderungen, da es nach einer großen Anzahl von Bestimmungen genan dasselbe Gewicht aufwies wie vorher.

Bei Beschickung des Verbrennungsofens führt man zuerst (wenn eine Marsofentype vorliegt) von der einen Seite das Thermoelement in das Schutzrohr ein und schließt das Rohr dort, dann schiebt man von der anderen Seite das Schiffchen $\left(7 \frac{1}{2} \mathrm{~cm}\right.$ lange lassen bis $\mathrm{zu} 5 \mathrm{~g}$ Nickel) bis zur Ofenmitte und läßt dann das Platinnetz tolgen, so daß dessen äußeres Ende mit dem Otenende abschneidet. Vor der Verwendung glüht man den Platindrahtzylinder gut aus. Aufmerksamkeit ist auch darauf $z u$ verwenden, das mit dem Drahtnetz keine an der Rohröffnung haftenden, vom Kautschukstopfen stammenden Gummipartikel ins Rohrinnere gebracht werden.

Zur Durchführung der Verbrennung hält man 10-15 Minuten die Temperatur auf $1150-1200^{\circ}$ und lä8t dann nach Ausschalten des Stromes den Sauerstoff noch etwa zehn Minuten durchstreichen. Der Sauerstoffstrom kann während der ganzen Verbrennung gleichmäbig langsam durchgeleitet werden. Die Absorptionsröhren werden in der üblichen Anordnung verwendet. Da Nickel gewöhnlich schwefelbältig ist, wird auch ein U-Rohr mit trockener Chromsäure eingeschaltet. Nachstehend einige Resultate zum Vergleich mit den Ergebnisen der Chromschwefelsäuremethode.

\begin{tabular}{|c|c|c|c|c|}
\hline Probematerial & $\begin{array}{c}\text { Einwage in } \\
\mathrm{g}\end{array}$ & $\begin{array}{c}\text { Auswage in } \\
\mathrm{g}\end{array}$ & $\begin{array}{c}\text { Gehalt an } \\
\mathrm{C} \text { in } \\
\%\end{array}$ & $\begin{array}{c}\text { Nach dem } \\
\text { Chromschwefel- } \\
\text { säureverfahren } \\
\text { gelunden } \\
\text { (Mittel) } \%\end{array}$ \\
\hline $\begin{array}{l}\text { Kohlenstoff . } \\
\text { Nickel. . }\end{array}$ & $\begin{array}{l}1,0073 \\
1,0007\end{array}$ & $\begin{array}{l}0,0681 \\
0,0676\end{array}$ & $\begin{array}{l}1,84 \\
1,84\end{array}$ & 1,83 \\
\hline Nickel II . & $\begin{array}{l}5,000 \\
5,000\end{array}$ & $\begin{array}{l}0,0237 \\
0,0233\end{array}$ & $\begin{array}{l}0,129 \\
0,127\end{array}$ & 0,128 \\
\hline Nickel III. & $\begin{array}{l}4,738 \\
5,021\end{array}$ & $\begin{array}{l}0,0087 \\
0,0084\end{array}$ & $\begin{array}{l}0,050 \\
0,046\end{array}$ & 0,050 \\
\hline Gußnickel . & $\begin{array}{l}5,020 \\
5,012\end{array}$ & $\begin{array}{l}0,0181 \\
0,0188\end{array}$ & $\begin{array}{l}0,098 \\
0,102\end{array}$ & 0,098 \\
\hline
\end{tabular}

Die angeführten Zahlen zeigen, daß die Methode sehr genaue Werte ljefert. Sie kann ebensoschnell wie bei Eisen und Stahl durchgeführt werden. Fehlerhalte Ergebnisse sind bei Finhaltung der vorgenannten Bedingungen fast ausgeschlossen.

Es existieren noch einige andere Methoden zur Kohlenstoffbegtimmung in Nickel, z. B. Verbrennung nach Abscheidung des Kollenstoffs durch Zusammenochmelzen des Nickels mit Zinn, doch sahen wir von einer Uberprüfung aus dem Grunde ab, weil sie zur Erzielung genauer Resultate zu wenig Aussicht boten. [A. 212.]

\section{Sonderausstellung der Fachgruppe für che- misches Apparatewesen auf der Achema in Hamburg 1922.}

Von Dr. HaNS WOLFF.

(Eingeg. am 14.j9.: 1922)

Uer Gedanke, die Laboratoriumsgerătschaften zu normieren hat noch nicht la ge bei den beteiligten Kreisen FuB gefaBt. Auf der ejnen Seite sah man wohl ein, daß es von Nutzen sein müsse, wenn man die häufig gebrauchlen GetăBe und Apparaturen in stets gleichartiver Ausföhrung erhalten und obne langes Suchen etwa einen Teil einer proberen Apparatur obne wejteres ersetzen konnte, anderseits aber fürchtete man gerade in den Chemikerkreisen, also bei den Verbrauchern, daA die Arheit durch die Normalisierung beeinträchtigt, die Auswahl verkleinert und die Hilfsmittel beschrinkt wärden.

Nun ist eine gewisse Beschränkung allerdings nötig, jedoch nur insofern, als hisher eine Unzahl der verschiedenen Gebrauchsgegenstände wie Kolben, Bechergläser usw. existierte, die vielfach nur geringe Abweichungen voneinander aufwiesen. Verlangt man aber von den F. brikanten, und das ist $j a$ das $A$ und $O$ einer Normierung, daß er seine Frzelugnisse mit größtmöglicher Genauigkeit herst..llt, so mul man auch ihm einen Vorteil la + ien. Unu dieser Vorteil kann nur der sein. alles das aus $d+r$ Normierung fortzulassen, was im Massenverbrauch nicht unbedirgt erforderlich ist. Alle Àrten von Spezialapparaturen, soweit sie nicht etwa schon, wie der „Engler- 